\title{
ENTRE LE RÉCIT POUR LES MASSES ET LE MYTHE D'INTERNET, LES CROYANCES SUR LES RÉSEAUX SOCIAUX COMME JEU DE CO-CONSTRUCTION
}

\begin{abstract}
Karim Chibout ${ }^{1}$ et Martial Martin ${ }^{2}$
L'étude porte sur des croyances exposées par les utilisateurs via certains outils numériques, principalement les réseaux sociaux virtuels, les blogs et les forums, ayant cependant pour origine des produits culturels de masse. Elle s'intéressera au point d'articulation entre d'une part une communication de type topdown à des fins de promotion de biens culturels (marketing et storytelling) et d'autre part la production d'une culture commune (d'un culte commun ?) par les utilisateurs des technologies numériques (web 2.0). Nous nous intéressons par exemple au culte Jedi-iste (Star Wars) et aux croyances eschatologiques (2012).

Paradoxalement, les « fausses » (?) croyances exprimées évoquent les plaisirs fugaces de la fiction (willing suspension of disbelief) mais étendus aux pratiques sociales du quotidien; elles s'apparentent à un jeu, une manière de fuir les désordres de la réalité (climatiques, économiques, sociaux, politiques, religieux...) et, dans le même temps, sont fortement connectées à cette réalité désenchantée. Nous avions déjà entraperçu cette contradiction interne dans l'analyse des constructions
\end{abstract}

1 Karim Chibout est Maître de conférences en sciences de l'information et de la communication à l'Université de Strasbourg, IUT d'Haguenau.

2 Martial Martin est Maître de conférences en sciences de l'information et de la communicationà l'Université de Reims, IUT de Troyes.

Recherches en communication, $\mathrm{n}^{\circ} 38$ (2012). 
imaginaires « faniques » autour de l'univers des jeux en ligne multi-joueurs (Chibout \& Martin, 2009).

A l'heure où les textes fondateurs (livres saints, constitution, traditions...) et les certitudes universelles sont ébranlés (Lyotard), on feint de croire à des réalités inventées de toutes pièces (pour ne pas dire faites de bric et de broc). Les échanges virtuels portant sur les pseudo-croyances renvoient, en effet, à des co-constructions hybrides mêlant faits scientifiques, prophéties ésotériques, superstitions et religion(s) mondiales et transhistoriques (qui prennent sens dans une créolisation généralisée).

Très liées aux circonstances qui les ont vues naître ou dans lesquelles on les a diffusées ou partagées, ces croyances sont marquées par l'inconstance ou l'inconsistance. L'absence de profondeur des « convictions » est à l'image de la labilité et de la fluidité des relations et des conduites installées dans la vie virtuelle.

On sait ce que le terme « croyance », que l'on pourra concevoir globalement comme un « état mental qui porte à donner son assentiment à une certaine représentation, ou à porter un jugement dont la vérité objective n'est pas garantie et qui n'est pas accompagné d'un sentiment subjectif de certitude »(Engel, 1995, p. 10-11), recouvre comme variétés de degrés de garantie objective ou de confiance subjective, ce qu'il a de particulièrement polysémique : « croyance » peut simplement renvoyer à une opinion fausse ou douteuse, un préjugé, une illusion, un enchantement ou une superstition, ou bien, plus susceptible d'être vrais, à un soupçon, une présomption, une supposition, une estimation, une hypothèse ou une conjecture ; si le sentiment subjectif du vrai est fort, mais le fondement objectif faible, on aura affaire à des convictions, des doctrines ou des dogmes ; enfin, croire en quelqu'un ou quelque chose peut participer de la confiance, de la foi ou de la fidélité, lorsque la croyance se place au-delà ou dans un autre ordre que les données ou les garanties objectives. L'Internet rend compte de l'ensemble de ces postures dans leurs diversités : il existe, donc, un Web des certitudes et des engagements. Dans une étude précédente (Chibout \& Martin, 2009), nous nous étions attachés à l'écriture, la production et la diffusion de photos et de vidéos sur le Web par les militaires, portés par une foi indéfectible en leur pays. Force est de constater que la communauté de croyance qu'ils tissent dans le réseau à travers leurs blogs (milblogging. com) et les plates-formes de partage de photos et de vidéos (Youtube, 
Flickr) se construit en réaction aux médias traditionnels. Dans l'« infoguerre », la guerre d'information qui accompagne les conflits militaires, pointe l'importance, sur le Web, des groupes bien structurés (hors ligne) et fortement organisés (cellules de communication des armées, membres actuels de forces en présence en Irak ou Afghanistan, anciens combattants, associations de femmes de militaires d'un côté, « cellules terroristes » de l'autre) guidés par leur profonde conviction politique ou leur foi (que l'on pourra juger dévoyées) dans leur combat contre les médias « dominants » prétendument inféodés à leurs ennemis idéologiques, et détournant à cette fin les plates-formes de journalisme citoyen ou collaboratif. Ce n'est ni le type ou le degré de croyance qui nous intéressera ici, ni le type de rapport de stricte opposition aux industries de la communication et de la culture. En effet, à l'autre extrémité du spectre des croyances en ligne, se trouvent, en particulier dans les réseaux sociaux (en l'occurrence Facebook), des formes tout à fait particulières de " demi-croyances » co-construites par les producteurs de contenus culturels et les consommateurs utilisateurs de ces services en ligne. Ces croyances - que, par commodité, nous appellerons ici indifféremment " demi-croyances », " croyances faibles 》, « croyances ténues 》 ou « fausses croyances 》- participent d'un assentiment fragile et passager à un motif fictionnel issu d'une production des industries culturelles de masse, qui se construit en ligne à travers les interactions des usagers (dans le « devenir fan » d'une page, « devenir membre » d'un groupe ou encore le « j'aime/I like » pour manifester son accord à la publication d'un ami), un consentement tout à fait ponctuel et circonscrit à ce qui, bien qu'issu d'une fiction, sera tenu pour réel, vraisemblable ou seulement possible mais pourra presque aussitôt donner lieu à une prise de distance amusée (comme si l'on s'amusait à croire'), une adhésion largement déterminée par la fréquentation des réseaux sociaux qui est, en même temps, affirmation d'une appartenance à un groupe de croyance tout aussi éphémère. C'est à leur étude que nous nous consacrerons ici en deux temps, d'une certaine manière en contrepoint à l'analyse souvent privilégiée de l'expression de croyances fortes sur des supports indifférents, à travers l'examen de quelques cinq cents profils Facebook d'étudiants ou de diplômés en multimédia et d'une cinquantaine de pages publiques de groupes ou d'applications : d'abord avec l'idée de décrire, dans une

1 Voir, dans ce numéro, l'article de Frédéric Lambert. Arts et industries de la croyance : quand le langage fait son cinéma, p. 105 
approche sémio-pragmatique, les énoncés et les actes des internautes sociaux, ensuite dans la volonté de dégager des dominantes dans les motivations sociales et psychologiques des utilisateurs.

\section{Les modalités du réinvestissement des produits culturels de masse dans les croyances des réseaux sociaux}

Parmi les sites de réseaux sociaux, Facebook s'est imposé comme un service incontournable sur la toile, loin devant les précurseurs comme Copains d'avant en France ou LinkedIn, Tribe, Friendster... jusqu'à devenir récemment le service le plus consulté du Web devant Google et amener les analystes à prédire la fin des blogs (autre symbole du Web 2.0 et autre moyen de cristalliser en ligne les relations du quotidien) au profit de ce type de réseau. Parmi les nombreuses fonctionnalités proposées, la plus décisive, couplée à la (re)constitution d'un réseau d'« amis » (selon l'appellation fixée) et au partage d'informations (le profil) et de photos, nous semble être celle de microblogging (autour du changement de statut). C'est elle qui nourrit les « amitiés », à travers les billets postés par l'utilisateur sur son « mur »; comme le résume Éric Loret dans le quotidien Libération:

Pour devenir populaire, il faut écrire des trucs magnifiques dans un rectangle appelé « statut » et temporairement rempli d'un engageant « Exprimez-vous ». Une fois bien exprimé, on reçoit sa récompense (ou sa vexation) sous forme de commentaires. À savoir : plein de commentaires, c'est qu'on est aimé, pas de commentaire, c'est trop la lose [sic], on est virtuellement (donc désormais réellement) ostracisé. (Loret, 2010)

Au centre de ces statuts, il y a la nécessité de la trouvaille, de l'invention, du trait d'esprit ; comme on le disait autrefois, il s'agit de " bien rencontrer»; et l'on voit clairement quelle dimension sociale peut revêtir cette pratique discursive. Or, ce qui nous a frappé est la co-présence dans les statuts de références à l'actualité médiatique et aux univers et personnages des productions de l'industrie culturelle, comme si la nouvelle et la fiction avaient le même degré dans l'échelle des croyances des utilisateurs. 
L'éruption en 2010 du volcan islandais Eyjafjöll (qui possède de nombreux fans sur Facebook ${ }^{1}$ ) ne manqua pas de déchaîner une activité herméneutique délirante qui mit en relation les faits rapportés à la télévision (en particulier les images de la télévision britannique Skynews montrant un objet volant dans le nuage volcanique) et les produits les plus récents de l'industrie culturelle, en l'occurrence le film-catastrophe 2012 qui prenait comme point de départ le réveil des grands volcans (par exemple sur 2012, le blog de l'apocalypse ${ }^{2}$ ou 2012, apocalypse ou fin du monde ${ }^{3}$ ). Les billets sur les blogs et les réseaux sociaux font donc écho à des croyances faibles véhiculées par les médias traditionnels. Or, comme on le sait (Hall, 1973), des produits comme le film de Joël Schumacher prennent déjà en compte les attentes des récepteurs au stade même de la production et d'une certaine manière en se référant à des croyances sociales diffuses sur les civilisations perdues, la fin du monde ou les complots mondiaux ${ }^{4}$. Ils permettent aux consommateurs de co-construire le produit et les croyances que celui-ci pourrait éventuellement diffuser. Le marketing se sert d'ailleurs clairement de ces croyances comme terrain d'une communication virale sur le Web annonçant souvent énigmatiquement la mise sur le marché du produit ${ }^{5}$. Dans ce cadre, ce sont les internautes qui, autour de leurs activités interprétatives, participent d'un bouche-àoreille qui prépare la sortie du film. Mais l'activité des consommateurs ne peut être entièrement dirigée et elle marque toujours par sa grande diversité qui souligne l'exceptionnelle liberté et l'incroyable inventivité des spectateurs. Si, et c'est là une preuve du succès du film autant que de l'efficacité du marketing, les pages de « fans » autour de 2012 sont nombreuses, elles se distinguent par une adhésion plus ou moins grande au propos de l'œuvre et par les modalités de l'ironie, de la mise à distance ou du regard oblique. De très nombreux groupes sur Facebook s'intitulent « 2012 »; ils véhiculent parfois, dans la lignée du film, des rumeurs eschatologiques; ils s'opposent aussi parfois frontalement au film en lui opposant une forme d'orthodoxie religieuse ${ }^{6}$ :

1 http://www.facebook.com/VolcanicAshCloud?v=wall\&ref=ts

2 http://2012leblogdelapocalypse.blogspot.be

3 http://www.2012-apocalypse-ou-fin-du-monde.fr

4 http://fr.wikipedia.org/wiki/Pr\%C3\%A9dictions_pour_d\%C3\%A9cembre_ 2012

5 Pour préparer la sortie du film 2012, il s'agissait, par exemple de conforter des rumeurs sur la fin du monde à travers un canular ou une mystification, le faux site Internet d'un très fictif Institute for Human Continuity.

6 Ce qui esquisse une piste de travail sur les relations entre les demi-croyances et la 
At the moment, all over the world there are people worrying about the year 2012 [...] We have disinformation crawling all over the internet, rumors buzzing around, and a wide variety of people who claim to know what is going to happen in 2012 [...] Before you take that the wrong way though, remember that all that Apocalypse really means is that some large secret will be revealed to a mass of people. Over thousands of years, the term has been twisted, like many other words, to mean the complete annihilation of the human species, but all it means is change ${ }^{1}$.

D'autres opposent une franche incrédulité, comme le groupe «A wéé 2012 On é Dan la Merd ?! fuk se Ki Croive A ‘2012’ Psk moi ji croi $\mathrm{Pa} »$. Mais, le plus souvent, ils sont dans un rapport ambigu tout à la fois de participation au mythe véhiculé et de mise à distance :

- soit par la mise en concurrence avec une autre production culturelle ( Pas de fin du monde en 2012, car Marty McFly [i.e. le héros de Retour vers le futur] est allé en 2015!! »);

- soit encore par le jeu sur les différents niveaux de la narration, avec par exemple la confusion entre personnage et acteur ( CEUX QUI DORMIRONT CHEZ CHUCK NORRIS LE 21 DECEMBRE $2012 »)$; - soit par le recours au quotidien (« Je ne serai pas là pour la fin du monde en 2012, j'ai poney »).

Chuck Norris et les poneys font, en effet, partie d'un folklore numérique, largement bricolé à partir de fragments de la culture médiatique $^{2}$. Et, au sein de cette mythologie, Star Wars est l'une des productions culturelles les plus investies. Les pièces de fanart affichées sur les murs des groupes ou des individus (en particulier des montages photographiques) montrent combien les consommateurs sont sensibles à l'hypotexte mystique ou religieux de la célèbre saga de science-fiction, que l'on sait très influencée par l'enseignement du mythologue Joseph Campbell suivi à l'université par George Lucas. On trouvera, par exemple, sur les pages des différents groupes constitués autour de cette fiction, un montage substituant du Pape Jean-Paul II à Darth Vador dans une scène où ce dernier étrangle un dignitaire de

foi religieuse.

1 http://www.facebook.com/group.php?gid=35857016126\&ref=search\&s id=100000666343542.1367825556..1

2 Sur le folklore numérique et le phénomène des "mèmes", voir Lialina \& Espenscheid, 2009. 
l'Empire en utilisant « la Force ». Une légende accompagne l'image : « I find your lack of faith... disturbing ». Un autre montage juxtapose simplement des photos du Pape Joseph Ratzinger et de «l'Empereur » afin de rapprocher les traits de leurs visages. Si la foi est clairement en jeu dans ces bricolages ou ces récupérations, il est difficile d'assigner un sens précis à l'utilisation de ces images (reprises à de très multiples occasions comme le veut cette culture du mème numérique) : par-delà la dimension ludique évidente, s'agit-il de reléguer le culte catholique au rang d'une fiction? Ou à l'inverse, faut-il voir là une reconnaissance du culte de la Force comme religion ? Rappelons qu'au RoyaumeUni, le recensement de 2001 fait apparaitre $0,7 \%$ de " jedi-istes ${ }^{1}$ » parmi les croyants. Ce culte de la Force retenant particulièrement les consommateurs, les statuts Facebook font apparaître un recours à cette mystique au quotidien, comme parmi les groupes "Pas besoin de réviser la Force est avec moi », "Pretending to use the Force to open automatic doors », « $\mathrm{i}$ wish $\mathrm{i}$ had the force just so $\mathrm{i}$ dont have to get up to get something $»$.

C'est là ce qui différencie nettement ce type d'activité et le braconnage culturel (de Certeau, 1980 ; Jenkins, 1992) tel qu'il s'exprime dans de nombreuses fanfictions diffusées sur le Web. Par sa structure sérielle, une saga comme Star Wars se prête particulièrement à ce type d'écriture fanique : entre les films, les jeux vidéo et les courts métrages d'animation qui déclinent un même univers fictionnel, les consommateurs comblent les béances en investissant leur imaginaire (Martin, 2007). Mais, si ces développements fictionnels participent, comme l'exposition des demi-croyances sur les réseaux sociaux, d'une logique de réappropriation des biens culturels, ceux-ci s'en séparent dans la réaffirmation de leur caractère fictif ${ }^{2}$, alors que les statuts Facebook jouent d'une ambiguïté entre le réel et le fictif et esquissent une croyance changeante, labile, ténue, entre l'espace fictionnel et la prose du quotidien. Du fait de ces entrelacements, les internautes sociaux semblent instaurer un mode particulier de croyance : la suspension volontaire d'incrédulité (Coleridge, 1817, p. 6), jusque-là circonscrite

$1 \mathrm{http}: / / \mathrm{www} \cdot$ statistics.gov.uk/CCI/nugget.asp? ID $=297 \& \mathrm{Pos}=\& \mathrm{ColRank}=2 \& \mathrm{Ra}$ $\mathrm{nk}=1000$

2 Même si occasionnellement certaines fanfictions intègrent un double de leur auteur : c'est le genre de Mary Sue. Sur les typologies des fanfictions, voir Jenkins, 1992, p. 162 et http://www.manfromuncle.org/kristl.htm. 
à l'expérience de lecture ou de visionnage d'un film, semble sans cesse activée, désactivée puis réactivée sur un support tel que Facebook, qui sert à la fois au partage de contenus culturels (par exemple à travers les vidéos vers lesquelles renvoie le «mur » de l'utilisateur) et à la communication interpersonnelle (à travers la fonction « chat»). L'usager sera, donc, tour à tour, crédule ou sceptique, sans pouvoir trancher, car, sur les réseaux sociaux, divers programmes de croyance semblent coexister parallèlement (Veyne, 1983) sans forcément entrer en conflit, sans que les individus ne les vivent comme incompatibles, pourvu qu'ils permettent la plus grande audience possible, pourvu qu'ils créent momentanément le plus de « liens/links » possible ou pour être plus juste le plus de «I like » possible. Car, les réseaux sociaux sont à la fois supports et objets de croyances; le fantasme technologique le dispute aux fausses croyances exprimées : les lieux virtuels d'échanges sont perçus comme des lieux de communautés ouvertes et libres, des espaces d'expression élargis, avec les illusions de la République virtuelle participative et de l'amitié portées par celle du nombre d'internautes sociaux.

\section{Les déterminants des demi-croyances des réseaux sociaux}

Les demi-croyances que nous venons de décrire nous paraissent co-déterminées par des dispositions pour la déification de l'objet culturel, la défection par rapport à une réalité vécue comme désenchantée, la réification des univers fictionnels (leur réinvestissement dans le réel), et enfin, la réfection (la restauration d'une identité communautaire de confort).

La « fausse » croyance de déification laisse entrevoir une inversion des codes cultu(r)els. À l'origine, c'est le mythe qui est créateur de l'objet de culture (en premier lieu, objet cultuel). À l'inverse, aujourd'hui, c'est l'objet culturel qui crée le mythe, au point que « les communautés de croyance » idolâtrant des objets sans objet deviennent des communautés sans âme, sans dévotion, aussi éphémères et futiles que le sont leurs objets de culte. Les célébrités alliant beauté, richesse et amour inconditionnel du plus grand nombre, bénéficient d'une aura quasi divine dans les masses, emportées par la ferveur et l'admiration pour ces êtres surnaturels ${ }^{1}$.

1 Voir, dans ce numéro, la contribution d'Amélie Dalmazzo sur Michael Jackson. Le 
La «fausse » croyance de défection correspond, dans la vision postmoderne du rapport à la réalité, à une « mise en fiction du monde » (Augé, 1997) et de soi qui veut rompre avec un quotidien désenchanté. Le trop-plein d'un réel trop présent, trop pressant, trop pesant aussi, fait déborder les esprits vers un semblant d'irrationnel, un fictionnel apaisant, un jeu de croyance qui permet d'abdiquer cette réalité en créant volontairement et temporairement un trompe-l'œil salutaire. L'ère du précis, du techn(olog)ique, de l'efficacité s'accompagne chez l'individu d'un sentiment d'insaisissabilité du monde. Les textes à l'origine des grandes cultures et les certitudes universelles sont en déclin (Lyotard, 1979) ; les méta-récits fondateurs sont supplantés par des micro-récits de pure invention auxquels les individus feignent de croire dans le cadre de micro-cultures épisodiques. Evacuant de la conscience, en tout cas pour un temps, une réelle source de stress et de craintes, la fausse croyance relève d'une sorte de scotomisation ou de « subjonctivisation » du réel au travers du récit (Bruner, 2002) : l'internaute " subjonctivise », selon l'expression de Bruner, la réalité en laissant place à un spectre de possibles, jette un pont entre ce qui est établi et ce qui est virtuel, revit le récit médiatisé par sa propre mise en scène, sa propre mise en fiction. La demi-croyance constitue une alternative à la réalité, une résistance au réel dans la réinvention et la réappropriation, une expression de la quête du futile et du rejet du réel en tant qu'obstacle au bien-être. Un monde en crise a donné naissance à un « individu incertain » (Ehrenberg, 1995) qui se débat seul dans une "vie liquide » (Bauman, 2006), précaire, complexe. " [Cette] individualisation croissante appelle de l'assistance " (Ehrenberg, 1995, p. 194) ; le jeu et le récit font partie de ces aides à être (alcool, drogues, médicaments psychotropes, cyberespace et autres prothèses affectives), rejets de la réalité au profit du vertige, du simulacre, de la logique émotionnelle et onirique. La fausse croyance (tout comme son support virtuel de diffusion) s'apparente à un îlot salvateur, un asile " désaliénant », un lieu de repos pour qui est " fatigué d'être soi » (Ehrenberg, 1998). Cela impose ici de fuir en d'autres lieux et d'autres temps, de s'éloigner de la vraie vie par des digressions fictionnelles le temps d'un échange virtuel, d'un récit amusé. La croyance " fausse » s'inscrit dans un mouvement social qui a renoncé à l'engagement politique ou religieux et choisi le clanisme onirique, le faux-fuyant

rôle des internautes dans la co-construction de la croyance en Michael Jackson : du King of Pop à Wacko Jacko, p. 123. 
dans le faux-semblant en pérennisant le rêve éveillé. Les mythologies « mythomaniaques » et apaisantes prennent le pas sur des idéologies à l'agonie. On touche là au simulacre (Baudrillard, 1985), réalité simulée devenue plus réelle que le réel, simulacre qui a pour vocation de pallier la réalité complexe et triste, désenchantée. Cependant, paradoxalement, si les « fausses » croyances évoquées s'apparentent à un jeu, une manière de fuir les désordres de la réalité (climatiques, économiques, sociaux, politiques, religieux...), dans le même temps, elles sont dystopiques, car fortement empreintes de cette réalité désenchantée.

Le pseudo-croyant feint d'ignorer le monde mais ne rompt pas avec les sphères de la vie réelle. Ni mythe fondateur ni tout à fait mythe sans fondations, la " fausse » croyance de réification, exposée en ligne, navigue entre fictionnel et réel. Dans les croyances autour de 2012, le futur ante catastrophem relève de l'imaginaire et s'accorde fortement avec la réalité prégnante des dangers immédiats encourus par l'humanité (réchauffement climatique lié aux pollutions...). Le faux-croyant diffuse des dystopies, réifie une fiction comme pour exprimer sa «passion du triste » ou sa crainte du morbide ; le récit porte le sceau du drame simplifié et reflète l'incertitude pessimiste d'un futur sans lendemains qui chantent. Les histoires coécrites en ligne, comme toutes les histoires, " nous procurent des modèles du monde » (Bruner, 2002, p. 16). Tout en s'ouvrant à la création, elles restent contraintes par certains schémas narratifs et explicatifs qui ramènent au réel. Justifiant la demi-croyance en la replaçant dans un système de pensée plus large en cohérence avec des faits, des croyances fortes, des données scientifiques, le récit ludique prend la tournure d'un schéma interprétatif, d'une construction signifiante, par scientifisation ou pour le moins rationalisation du propos dans un souci de mise en cohérence : une démarche par la preuve qui colle incidemment le récit au réel. Cette réification de la fiction est présente dans d'autres pratiques sociales. Ainsi dénonce-t-on une réalité tragique en usant de la fiction : lors de manifestations relayées par les médias, une tribu indienne a utilisé l'animation 3D Avatar $^{1}$ pour sensibiliser l'opinion à des causes écologiques. Les manifestants étaient grimés de bleu et costumés à la manière de l'ethnie extraterrestre $\mathrm{Na}$ 'vi. La fiction prend place dans la réalité comme outil de communication à privilégier pour son efficacité

1 Cf. l'article de Frédéric Lambert dans ce numéro, p. 105. 
dans l'effet sur le destinataire. Dans ces pratiques culturelles à caractère ludique, le fictionnel s'invite à nouveau dans le réel, nous conduisant à la frontière entre réel et fictionnel, entre réalité sans illusions et chimères ludiques, dans une "zone d'indistinction », pour reprendre la formule d'Agamben (1998). En définitive, le fictionnel prend, au moyen de la croyance de réification, la forme d'un réel affaibli.

Enfin, la motivation identitaire transparaît également dans les échanges portant sur les croyances ténues. En partageant des perceptions, en unifiant des réalités éparses et foncièrement subjectives, le réseau social permet au récit de s'affirmer en « creuset » d'une microculture du virtuel parce que " partager des histoires » c'est " créer une communauté interprétative [...] une communauté culturelle. » (Bruner, 2002, p. 38). Facebook, comme lieu de divertissement réservé, comme espace de sous-culture, permet d'installer une communauté onirique, une communauté d'envies et de restaurer, pour qui s'y implique, une identité communautaire autour d'un objet culturel, fût-ce provisoirement. L'événement ou l'objet culturel crée et maintient le lien à lui seul dans un groupe ponctuel d'appartenance, le groupe d'une référence. À la différence de la croyance forte, relativement figée, la demi-croyance est faite d'éléments composites dont l'agencement n'est jamais stabilisé, parce qu'elle se renégocie sans cesse au contact des autres. Le collectif, mû par une pratique et un intérêt communs, utilise le réseau comme dispositif sociotechnique de coproduction de savoirs profanes. On discerne dans cette «pensée connectée 》 les enjeux psychosociaux de la communication (Lipiansky, 1992) : convaincre, donner une image, s'affirmer et singulièrement créer du lien autour de l'objet d'attention. L'identification à l'objet s'accompagne d'une identification à un groupe : l'événement culturel ou le récit de fiction construit la communauté qui reconstruit le récit ou l'événement. C'est dans la réciprocité de l'échange que se crée la croyance. La narration est co-construite et façonnée par les interlocuteurs au fur et à mesure des échanges ; c'est un récit à plusieurs, une " hallucination collective» dans laquelle chacun amplifie le phénomène par ses commentaires. Dans les pages de fans sur Facebook, l'expérience du récit s'érige en un référentiel d'identification, identification fondée sur un mode émotionnel. Elle renvoie au partage passionnel, «à une subjectivité commune » (Maffesoli, 1988, p. 111), à une croyance-jouissance, car les messages échangés «n'ont pas d'abord pour but de faire savoir, mais de faire jouir » (Legendre, 1982, p. 92). Les internautes sociaux recréent un 
spectacle fascinant pour revivre la charge émotionnelle de l'événement originel. Ce jeu collectif finit par engendrer une communauté de l'imaginaire qui prend appui sur « le partage social d'émotions » (Rimé, 2005). Les faits, métaphores et autres allégories censés, dans les croyances vraies, expliquer l'ordre du monde ne servent dans le cas présent qu'à magnifier, enjoliver, romancer davantage encore la fiction. On s'écarte sensiblement de la mythologie d'explication du réel pour tendre vers une mythomanie d'implication ouverte à l'imagination. Il nous paraît pertinent d'assimiler l'implication sociale en présence à ce que Berry (2006) a qualifié d' ' immersion anthropologique » dans le cadre des communautés de jeux en ligne. Faire durer la jouissance, tel est sans doute le maître-mot de cette pratique virtuelle, une fusion dans un événement collectif à l'image de ce que Bourdieu (1977) appelle " le goût barbare » (qui, à la distance critique, préfère l'implication, «l'immersion dans la singularité de l'œuvre » (p. 34)) ou de « la culture du pauvre » (Hoggart, 1957) et spécifiquement de «l'identification fanique $»(B e s s o n, 2007$, p. 11).

\section{Conclusion}

Au point d'articulation entre une communication de type top-down à des fins de promotion de biens culturels (marketing et storytelling) et la production d'une culture commune (d'un culte commun ?) par les utilisateurs des technologies numériques (Web 2.0), émergent des phénomènes particulièrement intéressants comme le culte Jedi-iste (Star Wars) ou la résurgence de croyances eschatologiques (2012). Paradoxalement, les «fausses » ( ?) croyances exprimées évoquent les plaisirs fugaces de la fiction mais étendus aux pratiques sociales du quotidien ; elles s'apparentent à un jeu, une manière de fuir les désordres de la réalité (climatiques, économiques, sociaux, politiques, religieux...) et, dans le même temps, sont fortement connectées à cette réalité désenchantée. À l'heure où les certitudes universelles sont ébranlées, on feint de croire à des réalités inventées « de toutes pièces » (pour ne pas dire faites de bric et de broc). Les échanges virtuels portant sur les pseudo-croyances renvoient, en effet, à des co-constructions hybrides mêlant faits scientifiques, prophéties ésotériques, superstitions et religions mondiales et transhistoriques (qui prennent sens dans une « créolisation » généralisée). Très liées aux circonstances qui les ont vues naître ou dans lesquelles on les a diffusées ou partagées, ces croyances sont marquées par l'inconstance ou l'inconsistance. L'absence de 
profondeur des « convictions » est à l'image de la labilité et de la fluidité des relations et des conduites installées dans la vie virtuelle.

\section{Références}

Agamben, (1998). Ce qui reste d'Auschwitz: l'archive et le témoin. Paris: Éd. Payot et Rivages.

Augé, M. (1997). La guerre des rêves : exercices d'ethno-fiction. Paris : Seuil.

Baudrillard, J. (1985). Simulacres et simulation. Paris : Galilée.

Bauman, Z. (2006). La vie liquide. Paris : Éd. du Rouergue.

Berry, V. (2006). Immersion dans un monde virtuel : jeux vidéo, communautés et apprentissages. Observatoire des mondes numériques en sciences humaines. Disponible à http://www.omnsh.org/spip.php?article99.

Besson, A. (2007). La Fantasy. Paris : Klincksieck.

Bourdieu, P. (1977). La distinction : critique sociale du jugement du goût. Paris : Minuit. Bruner, J. (2002). Pourquoi nous racontons-nous des histoires? Paris : Retz \& Pocket. Certeau, M. de (1980). Arts de faire. Paris : Gallimard \& Folio Essais.

Chibout, K., \& Martin M. (2009). Militaires en mobilité : Web 2.0 et construction de l'identité. Dans F. Dervin \& Y. Abbas (Éd.), Technologies numériques du soi et (co-) constructions identitaires. (pp. 33-57). Paris : L'Harmattan.

Coleridge, S. T. (1817). Biographia Literaria. Dans The Collected Works : t. VII, vol. 2. Princeton : Princeton University Press.

Ehrenberg, A. (1995). L'individu incertain. Paris : Hachette Littératures.

Ehrenberg, A. (1998). La fatigue d'être soi, dépression et société. Paris : Éditions Odile Jacob.

Engel, P. (1995). Croyances. Dans D. Kambouchner (Éd.), Notions de philosophie II. (pp. 9-101). Paris : Gallimard \& Folio.

Hall, S. (1994[1973]). Codage, décodage. Réseaux, 68, 27-39.

Hoggart, R. (1957). La culture du pauvre. Paris : Minuit.

Jenkins, H. (1992). Textual poachers. New York : Routledge.

Legendre, P. (1982). Jouir du pouvoir : traité de bureaucratie patriote. Paris : Minuit.

Lialina, O., \& Espenscheid, D. (Éd.). (2009). Digital floklore. Stuttgart : Merz \& Solitude.

Lipiansky, E.-M. (1992). Identité et communication. Paris : Presses universitaires de France.

Loret, E. (18 février 2010). Facebook invente l'esthétique m'as-tu-lu. Libération. Disponible à : http://www.liberation.fr/livres/0101619974-facebook-invente-1esthetique-m-as-tu-lu.

Lyotard, J.-F. (1979). La condition postmoderne. Paris : Minuit.

Maffesoli, M. (1988). Le temps des tribus : le déclin de l'individualisme dans les sociétés de masse. Paris : Méridiens-Klincksieck.

Martin, M. (2007). Les séries télévisées sur Internet : le cas des « fanfictions ». Médiamorphoses, Hors Série n³, 186-189. 
Martin, M. (2008). L'irruption d'une nouvelle forme narrative : les « alternate reality games ». Dans A. Saemmer \& M. Maza (Éd.), e-formes. (pp. 45-58). Saint-Etienne : Presses Universitaires de Saint-Etienne.

Proulx, S. (2002). Trajectoires d'usages des technologies de communication : les formes d'appropriation d'une culture numérique comme enjeu d'une société du savoir. Annales des télécommunications, t. 57, 3-4, 180-189.

Rimé, B. (2005). Le partage social des émotions. Paris : Presses universitaires de France.

Veyne, P. (1983). Les Grecs ont-ils cru à leurs mythes? Paris : Seuil.

Weissberg, J.-L. (1999). Présences à distance : déplacements virtuels et réseaux numériques. Paris : L'Harmattan. 